(C) 1981. The Genetical Society of Great Britain

\title{
WORKER-QUEEN CONFLICT AND SEX RATIO THEORY IN SOCIAL HYMENOPTERA
}

\author{
M. G. BULMER and P. D. TAYLOR \\ Department of Biomathematics, Pusey Street, Oxford; Department of Mathematics, \\ Queen's University, Kingston, Ontario
}

Received 22.i.81

\section{SUMMARY}

\begin{abstract}
There is a conflict of interest between the queen and her worker-daughters in social hymenoptera over the ratio of investment in male and female reproductives. In the absence of worker-laying and inbreeding, the queen prefers a $1: 1$ (male: female) investment ratio, whereas the workers prefer a $1: 3$ ratio. Trivers and Hare (1976) suggest that the workers will win this conflict because they control the allocation of care to the young, but this argument ignores the fact that the queen controls the numbers of haploid and diploid eggs laid, and that the workers must operate within this constraint. We have investigated two theoretical models of this situation. We conclude that the queen may have considerable control over the investment ratio; two factors which may act in favour of worker control are a high cost of producing a new queen rather than a worker and the possibility of making a variable investment in a new queen with a concomitant variation in her fitness.
\end{abstract}

\section{INTRODUCTION}

IN an influential paper, Trivers and Hare (1976) have discussed the conflict of interest between the queen and her worker-daughters over the production of reproductives in colonies of social hymenoptera. This conflict has two interacting aspects-conflict over which party will produce male eggs and conflict over the ratio of investment in male and female reproductives. What determines which party will win these conflicts? Trivers and Hare (1976) argue plausibly that the queen should win the conflict over the production of male eggs in monogynous, perennial colonies (colonies such as those of many ants which have a single queen and which last for many years). The reason is that there is a strong asymmetry of interest in a physical contest between the queen and a worker; a worker who kills the queen will suffer a large loss of inclusive fitness because the colony can produce no more workers, whereas a queen who kills a worker will hardly notice the effect on her inclusive fitness. Thus the queen can destroy worker-laid eggs without fear of retaliation, and one would expect all eggs, haploid as well as diploid, to be laid by the queen. Worker-laying is in fact rare in monogynous, perennial colonies, except after the death of the queen.

Trivers and Hare (1976) then argue that, if all males are queenproduced, the workers will maximise their inclusive fitness by investing three times as much effort in rearing female as in rearing male reproductives (because they are related three times as closely to their sisters as to their brothers as a result of haplo-diploidy), whereas the queen would prefer an equal investment in male and female reproductives (because she is equally closely related to her sons as to her daughters). The above authors suggest that this conflict of interest is likely to be won by the workers, who care 
for the young and can therefore determine how this care is allocated. They write: "Since in ants workers feed and care for the reproductives from the time the reproductives are laid as eggs until they leave the nest as adults and since there are usually hundreds of workers (or more) per queen, it is difficult to see how an ant queen could prevent her daughters from producing almost the ratio of investment that maximises the workers' inclusive fitness". They therefore conclude that in monogynous, perennial colonies of ants the investment ratio in reproductives should be biased in favour of females by a margin of $3: 1$, and they present data to show that this is in fact the case, though there has been some controversy over both the statistical analysis and the interpretation of these data (Alexander and Sherman, 1977; Macnair, 1978; Forsyth, 1980; Taylor, 1981).

In this paper we wish to consider the theoretical question, whether the queen or the workers ought to win the conflict over the investment ratio, or whether some compromise between them should be reached. It seems to us that Trivers and Hare (1976) have overlooked one important weapon possessed by the queen, that in the absence of worker-laying she determines the numbers of haploid and diploid eggs laid; the workers can then invest their resources in making males from haploid eggs or in making either queens or new workers from diploid eggs, subject to the numbers of these eggs laid by the queen and to the total resources available for investment. In the next section we shall construct and analyse a model which incorporates these ideas.

\section{A model For a PERENNiAl COLONy}

Consider a haplodiploid social insect with the following life history. The population is composed of a large number of colonies, each of which has a single queen who lays all the eggs. Each summer the colonies produce male and female reproductives who mate at random within the population; mated queens (who have mated once only) try to found new colonies. Established colonies have a chance $p$ of surviving from one summer to the next, irrespective of their age or size; in the numerical calculations we shall take the survival rate as $p=0 \cdot 8$, so that the average lifetime of a colony is five years.

Suppose that in its $t$ th summer a colony has resources $R_{t}$ available for making new individuals. The queen can determine how many eggs are laid, and she chooses to lay $D_{t}$ diploid eggs and $H_{t}$ haploid eggs, with no constraints except that $D_{t} \geqq 0, H_{t} \geqq 0$. (We are assuming that constraints on colony growth and the production of reproductives are provided by the ability of the workers to rear larvae, embodied in $R_{t}$, rather than by the ability of the queen to lay eggs.) The workers can now allocate the resources $R_{t}$ among these eggs, and they choose to rear $W_{t}$ new workers, $Q_{t}$ queens and $M_{t}$ males, subject to the constraints that $W_{t} \geqq 0, Q_{t} \geqq 0, M_{t} \geqq 0$, and that

$$
\begin{gathered}
W_{t}+Q_{t} \leqq D_{t} \\
M_{t} \leqq H_{t} \\
W_{t}+\alpha Q_{t}+\beta M_{t} \leqq R_{t}
\end{gathered}
$$

(1(iii)) is the resource constraint, which assumes that $\alpha$ is the cost of a queen and $\beta$ the cost of a male, both relative to the cost of a new worker, 
taken as 1 unit. If the colony survives till the next summer, we suppose that the resources available then will depend only on $W_{t}$ and will have the form

$$
R_{t+1}=\left[1-\exp \left(-\frac{\mu}{K} W_{t}\right)\right] K
$$

The parameter $\mu$ is the maximal growth rate of the colony if it is small and devotes all its resources to growth rather than reproduction. In the numerical calculations we shall take $\mu=10$. The parameter $K$ determines the maximum size of the colony; its value will not affect the form of the optimal strategy.

Before proceeding with a detailed analysis of this model it may be helpful to indicate what sort of solution to expect. If we put $p=0$ so that the colony never survives from one year to the next, we would have a model for an annual colony. In this case there is no point in producing any new workers, and the queen can enforce the sex ratio she wants by limiting the supply of diploid eggs (all of which the workers will make into queens) and forcing them to use their remaining resources in rearing haploid eggs into males. However, a realistic model of an annual colony should take into account the possibility of worker-laying and the fact that there are several generations of workers in a year; a model taking these complications into account will be considered elsewhere (Bulmer, 1981).

In the present paper we consider a model for a perennial colony with $p$ nearer 1 than 0 ; it is plausible to ignore worker-laying in this case, as explained in the Introduction, and also to concentrate on a single point of time in each year at which colonies release reproductives. In this situation it will be in the interests of both parties to produce some workers each year to carry on the colony next year, if it survives. This fact gives the workers some influence on the outcome of the contest over the sex ratio among reproductives; the queen cannot afford to limit the supply of diploid eggs too severely because that would limit the production of new workers and would entail too low an amount of resource the next year. How can we calculate the optimal strategies for the queen and for the workers?

A strategy for the queen $\left(S_{Q}\right)$ is the choice of two non-negative functions $D(R)$ and $H(R)$ specifying the numbers of diploid and haploid eggs to lay when the resources available to rear them are $R$. A strategy for the workers $\left(S_{W}\right)$ is the choice of three non-negative functions $W, M$ and $Q$, depending on $D, H$ and $R$, satisfying the constraints (1) and specifying the numbers of workers, males and queens to rear given $D, H$ and $R$. If the queen's and the workers' strategies in a particular colony are known, together with $R_{1}$, the resources available in its first year of life, its subsequent history in any year in which it has survived can be calculated. Hence we can find the expected numbers of queens and males produced during the lifetime of that colony, given by

$$
\begin{aligned}
\hat{Q} & =\sum Q_{t} p^{t} \\
\hat{M} & =\sum M_{t} p^{\prime} .
\end{aligned}
$$

(In giving equal weight to the numbers of reproductives produced in different years, we are presupposing that the number of colonies in the population and the numbers of reproductives they produce each year are 
constant. This is likely to be true at equilibrium, so that this approach will give the correct equilibrium strategies, assuming that they exist. In the numerical calculations we shall take $R_{1}=0 \cdot 01 K$.)

Both the queen and the workers want to maximise the Expected number of reproductives produced during the lifetime of the colony, but they attach different weights to queens and males. If $s$ is the population sex ratio, the queen will try to maximise

$$
F_{Q}=s \hat{Q}+(1-s) \hat{M}
$$

while the workers will try to maximise

$$
F_{W}=3 s \hat{Q}+(1-s) \hat{M} \text {. }
$$

$F_{Q}$ and $F_{W}$ will be called the queen's and the workers' objective functions respectively. A justification of these objective functions is given in the Appendix.

Both $F_{Q}$ and $F_{W}$ are each functions of both $S_{Q}$ and $S_{W}$. If the queen tries to find a strategy to maximise $F_{Q}$, while the workers try to find a strategy to maximise $F_{W}$, they will end up with a pair of strategies $\left(S_{O}^{*}, S_{W}^{*}\right)$ which form an evolutionarily stable strategy (ESS) such that

$$
\begin{array}{ll}
F_{Q}\left(S_{Q}, S_{W}^{*}\right) \leqq F_{Q}\left(S_{Q}^{*}, S_{W}^{*}\right) & \text { for all } S_{Q} \\
F_{W}\left(S_{Q}^{*}, S_{W}\right) \leqq F_{W}\left(S_{Q}^{*}, S_{W}^{*}\right) & \text { for all } S_{W} .
\end{array}
$$

Denote by $\hat{Q}^{*}$ and $\hat{M}^{*}$ the Expected numbers of queens and males produced by a colony employing this ESS, and let the sex ratio in these colonies be

$$
r^{*}=\hat{M}^{*} /\left(\hat{M}^{*}+\hat{Q}^{*}\right) .
$$

Thus $r^{*}$ is the sex ratio evolved in response to the population sex ratio $s$, which has been regarded as constant. If we plot $r^{*}$ as a function of $s$ we shall obtain a decreasing function. The evolutionarily stable sex ratio is the value of $s$ for which $r^{*}(s)=s$.

If the population sex ratio is $s$, the corresponding investment ratio in males is

$$
\sigma=\beta s /[\beta s+\alpha(1-s)]
$$

For investment ratios between worker control $\left(\sigma=\frac{1}{4}\right)$ and queen control $\left(\sigma=\frac{1}{2}\right)$, which is the range of values of interest, the queen will prefer a unit of resource to be invested in making males rather then queens (if it is to be used in making reproductives), whereas the workers would rather invest it in queens than males. It is therefore unlikely that it would be to the queen's advantage to restrict the supply of haploid eggs. To simplify the analysis we shall therefore assume that the queen lays as many haploid eggs as the workers will rear, so that the constraint (1(ii)) can be ignored. The power of the queen lies in her ability to restrict the number of diploid eggs.

For a fixed value of $s$ the ESS $\left(S_{Q}^{*}, S_{W}^{*}\right)$ was calculated by the following dynamic programming approach. Suppose that, if the colony miraculously survives for 1000 years, it will then devote all its resources to reproductives in some given ratio of males and females and die. We can calculate the equilibrium strategies in previous years by backward iteration. Suppose 
that the equilibrium strategy in year $1000-n$ has been found; write $\hat{Q}_{n}^{*}(R)$ and $\hat{M}_{n}^{*}(R)$ for the expected numbers of queens and males produced from that year on, if it has resources $R$ in that year. If the colony has resources $R$ in year $1000-(n+1)$ and produces $W$ workers, $Q$ queens and $M$ males in that year, the resources available in the following year if it survives will be

$$
\bar{R}=\left[1-\exp \left(-\frac{\mu}{K} W\right)\right] K,
$$

and the Expected numbers of reproductives produced from year 1000$(n+1)$ onwards will be

$$
\begin{aligned}
\hat{Q}_{n+1} & =Q+p \hat{Q}_{n}^{*}(\bar{R}) \\
\hat{M}_{n+1} & =M+p \hat{M}_{n}^{*}(\bar{R}) .
\end{aligned}
$$

For fixed $D$ we can now find the values of $W, Q$ and $M$ satisfying (1) which maximise the workers' objective function; using these values and varying $D$ we can find the value of $D$ which maximises the queen's objective function, assuming that the workers will maximise their objective function. Hence the equilibrium strategy in year $1000-(n+1)$ can be computed. As we proceed backwards and $n$ increases, the effect of imposing the arbitrary strategy in year 1000 will disappear and the solution will converge to the ESS $\left(S_{Q}^{*}, S_{W}^{*}\right)$ for the original problem.

Equilibrium strategies have been calculated in this way for different values of $s$ and hence the equilibrium sex ratio satisfying $r^{*}(s)=s$ has been found with parameter values $p=0 \cdot 8, \mu=10, R_{1}=0 \cdot 01 \mathrm{~K}$. The equilibrium investment ratios, as defined in (7), are shown in table 1 for different values of $\alpha$, the cost of a queen relative to that of a worker; the results do not depend on $\beta$, the cost of a male. With queen control we expect $\sigma=0.5$ and with worker control $\sigma=0 \cdot 25$. Thus there is a large measure of queen control unless $\alpha$ is very large. It is intuitively clear why queen control becomes weaker as $\alpha$ increases since there is more scope for workers to react to limitation of diploid eggs by investing more resources in making queens; it is surprising (at least to us) that the degree of queen control is as high as it is.

The computer calculations required to produce the results in table 1 were costly, and it was unfortunately not possible to repeat them for other values of the parameters, but we feel that the results are likely to be qualitatively correct for most biologically plausible parameter values. We have already noted that $K$ is a scale parameter which will not affect the sex ratio. Provided that $R_{1}$, the size of a newly-formed colony, is small compared with $K$, and that $\mu$, the maximum colony growth rate, is reasonably large, the colony will devote all its resources to producing workers in its first year or two of life; only when the colony has reached a size at which its actual growth rate has fallen to some critical level will it produce

TABLE 1

Equilibrium investment ratio in males as a function of the cost of a queen

$\begin{array}{lcccc}\text { Relative cost of a queen }(\alpha) & 1 & 2 & 5 & 10 \\ \text { Investment ratio }(\sigma) & 0.499 & 0.478 & 0.443 & 0.385\end{array}$


reproductives, so that its behaviour in the reproductive phase will be insensitive to $R_{1}$ and $\mu$, which only affect its initial behaviour in the ergonomic phase. The value of $p$, the survival rate, will certainly affect the power of the queen to control the sex ratio, but we have chosen a value of $p=0.8$ which seems reasonable for perennial colonies.

\section{THE EFFECT OF VARIABLE COSTS}

In the previous section we assumed that there were fixed costs of producing workers, queens and males. In fact the workers might have a choice in the amount invested in a particular individual; for example, they might be able to produce a queen of high fitness at high cost or a queen of lower fitness at lower cost. In this section we shall investigate whether such flexibility would give the workers more control over the investment ratio. This idea was suggested to us by the work of Maynard Smith (1980) on the theory of sexual investment in diploid species.

It is impractical to introduce this complication into the already complicated model of the last section, and we shall therefore discuss it in the context of a simpler situation. Consider an annual haplodiploid social insect in which the colony produces reproductives once only at the end of the year and then dies. Suppose that in the reproductive phase the queen lays $D$ diploid eggs and an unlimited supply of haploid eggs and the workers have resources $R$ available for making reproductives, where we choose units so that a male requires 1 unit of the resource. The workers must choose the number $t$ of units to invest in each queen, with the constraint $t D \leqq R$. We suppose that an investment of $t$ confers a fitness of $g(t)$ upon a new queen.

This fitness can be most simply interpreted by supposing that some value $t_{0}$ corresponds to a "standard" queen, and for other values of $t, g(t)$ measures expected genetic contribution to the next generation relative to a standard queen. So the output of the colony is $Q=D g(t)$ standard queens and $M=R-t D$ males.

Given $D$, the workers should choose $t$ to maximise $Q^{3} M$. If we denote by $t^{*}=t^{*}(D)$ the maximising value of $t$, then the queen should choose $D$ to maximise $Q M$, where $Q$ and $M$ are evaluated at $t=t^{*}(D)$. The justification for this is contained in the Appendix, if "queen" is replaced by "standard queen".

We first solve the workers' problem for fixed $D$. Equating to zero the derivative of $Q^{3} M$ with respect to $t$ we get

$$
3 M g^{\prime}(t)=Q
$$

where $g^{\prime}$ denotes the derivative of $g$. Equation (8) with $t=t^{*}$, gives $t^{*}$ implicitly as a function of $D$. Now we solve the queen's problem by equating to zero the derivative of $Q M=D g\left(t^{*}\right)\left(R-t^{*} D\right)$ with respect to $D$, where $t^{*}$ must be treated as a function of $D$. We get

$$
\frac{d t^{*}}{d D}\left(D g^{\prime}\left(t^{*}\right) M-D Q\right)=t^{*} Q-M g\left(t^{*}\right)
$$

The simplest way to eliminate $d t^{*} / d D$ from (9) is to regard (8) as giving $D$ as a function of $t^{*}$, and differentiate with respect to $t$ to get an expression 
for $d D / d t^{*}=\left(d t^{*} / d D\right)^{-1}$. If this expression is incorporated into (9) we get, eventually, the equilibrium condition

$$
g g^{\prime \prime}\left(3 g^{\prime}-g / t\right)=6\left(g^{\prime}\right)^{2}\left(g^{\prime}-g / t\right)
$$

whose solution $t$ is the value of $t^{*}$. If we assume $g$ and $g^{\prime}$ are positive while $g^{\prime \prime}$ is negative, then (10) requires that at $t=t^{*}, g / t$ lie between $g^{\prime}$ and $3 g^{\prime}$. Now the ratio of investment in males to investment in queens is

$$
\frac{M}{t D}=\frac{g}{3 t g^{\prime}}
$$

by $(8)$, so that (10) requires a ratio between $1: 3$ and $1: 1$.

The situation is illustrated in fig. 1 where a typical $g$ curve is drawn. The boundaries for $t^{*}, g / t=g^{\prime}$ and $g / t=3 g^{\prime}$ are shown. The quantity $g / t$ is the ratio of the fitness of a queen to the original investment, and is seen from the graph to be maximised when $g / t=g^{\prime}$. Thus the $1: 3$ ratio is the one at which queen production is most efficient.

As an example we take one of the investment functions considered by Maynard Smith (1980):

$$
g(t)=c t^{a} /\left(b+t^{a}\right)
$$

with parameters $a, b$, and $c>0$. If $a>1$, the graph of $g$ has the form given in fig. 1. The equilibrium condition (10) for this function is

$$
t^{a}=b(a-1)(3 a+1) /(a+1)
$$

and the ratio (11) becomes

$$
\frac{g}{3 \operatorname{tg}^{\prime}}=\frac{3 a-1}{3 a+3}
$$

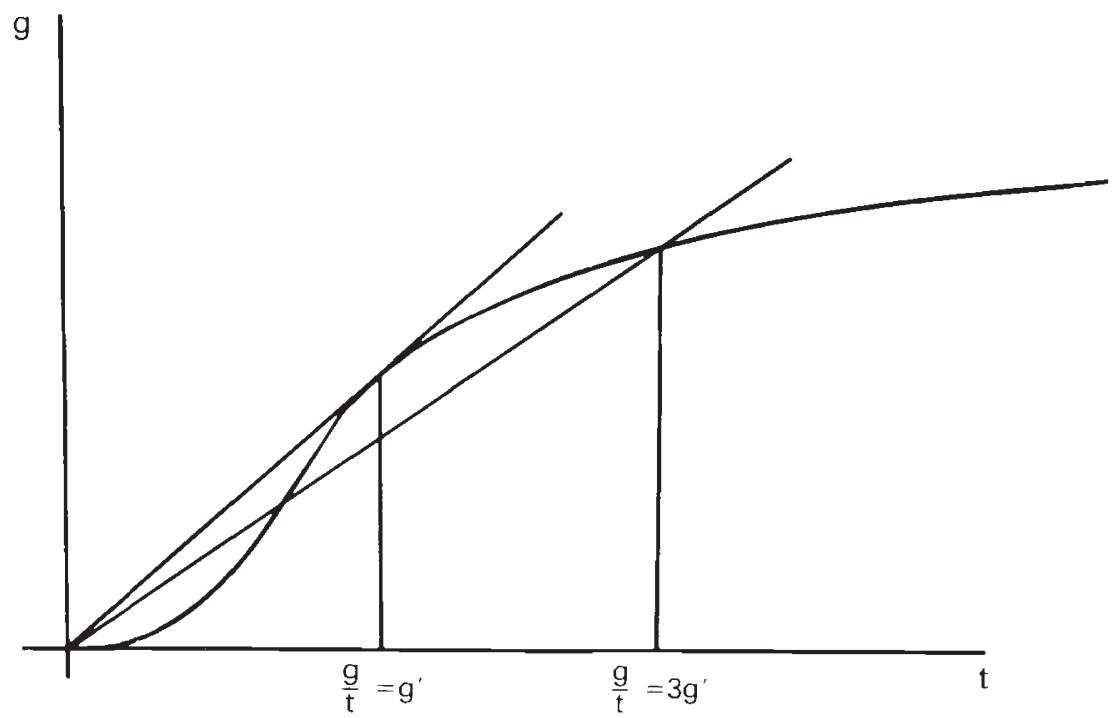

FIG. 1.-Queen's fitness $g$ as a function of investment $t$. The equilibrium value of $t$ must lie between the points $g / t=g^{\prime}$ and $g / t=3 g^{\prime}$. The first point gives an investment (male : female) ratio of $1: 3$ and the second a ratio of $1: 1$. 
which goes from the workers' preferred ratio of $1: 3$ when $a$ is close to 1 to the queen's preferred ratio of $1: 1$ for large $a$. To see why this is reasonable observe that the effect of increasing $a$ on the graph of $g$ is to make the curve rise more quickly to the asymptote. For example the family of functions $g(t)=c t^{a} /\left(9+t^{a}\right)$ all take the value $c / 10$ at $t=1$. They attain the value $9 c / 10$ at $t=9$ if $a=2$, and at $t=3$ if $a=4$. Then the amount of extra investment required to raise a queen's fitness from $\frac{1}{i \overline{0}}$ to $\frac{9}{10}$ of its maximum value is 8 units when $a=2$ and only 2 units when $a=4$. In the second case the workers have less flexibility and hence less control.

\section{Discussion}

There is a conflict of interest between the queen and her workerdaughters in social hymenoptera over the ratio of investment in male and female reproductives. If a single non-inbred queen lays all the eggs, she prefers a 1:1 (male: female) investment ratio, whereas the workers prefer a $1: 3$ ratio (since they are three times as closely related to their sisters as to their brothers under haplodiploidy). In this paper we have undertaken a theoretical analysis of this conflict, taking into account that the queen controls the numbers of haploid and diploid eggs laid, while the workers control the investment of resources in making males from haploid eggs or in making either queens or new workers from diploid eggs. The two models considered are idealised and ignore many of the complexities in the natural history of any real species, but we believe that they throw light on some of the factors which are important in resolving the outcome of this conflict of interest.

In section 2 we have considered a simple model of a perennial colony with fixed costs of producing workers, males and queens; we have found the evolutionarily stable sex ratio arising from the balance between the interests of the two parties by a dynamic programming approach. The equilibrium investment ratio (male: female) depends only on the relative cost of producing a queen rather than a worker from a diploid egg. As shown in table 1 , the investment ratio is much closer to the $1: 1$ ratio preferred by the queen than to the $1: 3$ ratio preferred by the workers unless the cost of producing a queen is very high.

In section 3 we have considered whether the possibility of producing a queen of high fitness at high cost or a queen of lower fitness at lower cost will give the workers greater control over the investment ratio. To simplify the analysis we have considered an annual colony which produces reproductives once only at the end of the year and then dies. Under this model the queen has complete control over the investment ratio with fixed costs of producing male and female reproductives, but we find that allowing a variable investment gives an appreciable degree of control to the workers if the sensitive range over which fitness responds to variations in investment is large. Putting the results of sections 2 and 3 together, it seems likely that in a perennial colony, a high average cost of a queen and the possibility of variable investment in individual queens would both act in favour of worker control over the investment ratio.

It has been assumed throughout this paper that there is no workerlaying, so that our conclusions are more relevant to perennial colonies, in which worker-laying is likely to be unimportant for reasons discussed in 
the Introduction, than to annual colonies (notwithstanding that the model in section 3 presupposes an annual colony). Consideration of the conflict of interest over worker-laying would add a new dimension of difficulty to the analysis.

Finally, we may place the queen-worker conflict over sex ratio in social hymenoptera in a more general setting by noting its similarity to the mother-daughter conflict over sex ratio in gregarious hymenopteran parasitoids discussed by Pickering (1980). In the latter case there is competition for host resources between sibs which through kin selection in mixed broods will give an advantage to females over males. Pickering (1980) suggests that mothers tend to lay broods with a preponderance of one sex or the other as a strategy to avoid competition between the sexes which gives rise to a distorted investment ratio.

\section{REFERENCES}

ALEXANDER, R. D., AND SHERMAN, P. W. 1977. Local mate competition and parental investment in social insects. Science, 196, 494-500.

BULMER, M. G. 1981. Worker-queen conflict in annual social hymenoptera. J. theor. Biol. In press.

Charnov, E. L. 1978. Sex ratio selection in eusocial hymenoptera. Amer. Nat., 112, 317-326. FORSYTH, A. 1980. Sex ratio and parental investment in an ant population. (Submitted to Evolution.)

MACNAIR, M. R. 1978. An ESS for the sex ratio in animals, with particular reference to the social hymenoptera. J. theor. Biol, 70, 449-459.

MAYNARD SMITH, J, 1980. A new theory of sexual investment. Behav. Ecol. Sociobiol., 7, 247-251.

PICKERING, J. 1980. Larval competition and brood sex ratios in the gregarious parasitoid Pachysomoides stupidus. Nature, 283, 291-292.

TAYLOR, P. D. 1981. Sex ratio compensation in ant populations. (Submitted to Evolution.)

TAYLOR, P. D., AND BULMER, M. G. 1980. Local mate competition and the sex ratio. $J$. theor. Biol., 86, 409-419.

TRIVERS, R. L., AND HARE, H., 1976. Haplodiploidy and the evolution of social insects. Science, 191, 249-263.

\section{APPENDIX}

The standard technique for calculating equilibrium sex ratios (Charnov, 1978; Taylor and Bulmer, 1980) is to suppose that sex ratio of offspring is controlled by a gene at a single locus. Assume all members of the population have the wild-type allele $T^{*}$ except a few mutant individuals who carry an alternative allele $T$. The sex ratio coded by $T^{*}$ is an ESS if every such $T$ has a growth rate (numbers of copies per generation) no greater than that for $T^{*}$.

If $T$ is rare and inbreeding is negligible we can ignore $T T$ individuals and assume there are two kinds of mutant individuals, $T T^{*}$ queens and $T$ males. If we let $x$ and $y$ denote the numbers of these mutant queens and males respectively in one generation then the corresponding numbers $\bar{x}$ and $\bar{y}$ one generation later (we assume discrete non-overlapping generations, but see Note 1) will be linearly related to $x$ and $y$ with a transition matrix $\boldsymbol{A}$ whose entries depend on the wild-type and mutant sex ratios. The first column of $\boldsymbol{A}$ is the mutant reproductive output of a mutant 
queen : numbers of mutant queen and mutant male offspring, and the second column is the mutant offspring output of a mutant male.

Suppose that $t$ parameterizes the colony's choices; a given $t$ corresponds to the production of $Q(t)$ queens and $M(t)$ males. We let $t^{*}$ be the parameter value coded by $T^{*}$, and $t$ be that coded by $T$.

If the queen has sex ratio control, the gene expresses itself only in the queen, and the transition matrix is

$$
\boldsymbol{A}_{Q}=\boldsymbol{A}_{Q}\left(t, t^{*}\right)=\left[\begin{array}{cc}
\frac{Q(t)}{2} & \frac{Q\left(t^{*}\right)}{M\left(t^{*}\right)} Q\left(t^{*}\right) \\
\frac{M(t)}{2} & 0
\end{array}\right]
$$

We have assumed mutant individuals always have normal mates, and each male can expect to have $Q\left(t^{*}\right) / M\left(t^{*}\right)$ mates. If the workers have sex ratio control the matrix is

$$
\boldsymbol{A}_{W}=\boldsymbol{A}_{W}\left(t, t^{*}\right)=\left[\begin{array}{cc}
\frac{Q(t)+Q\left(t^{*}\right)}{4} & \frac{Q\left(t^{*}\right)}{M\left(t^{*}\right)} Q(t) \\
\frac{M(t)+M\left(t^{*}\right)}{4} & 0
\end{array}\right]
$$

Notice that in a mutant-queen colony half the workers are mutant whereas in a colony fathered by a mutant drone all the workers are mutant. In the former case we assume each type of worker appropriates half the reproductive resources.

If we let $\lambda\left(t, t^{*}\right)$ be the dominant eigenvalue of $\boldsymbol{A}\left(t, t^{*}\right)$ then $t^{*}$ is the ESS value if $\lambda\left(t^{*}, t^{*}\right) \geqq \lambda\left(t, t^{*}\right)$ for all $t$, which implies

$$
\frac{\partial \lambda}{\partial t}\left(t^{*}, t^{*}\right)=0
$$

There is a nice condition equivalent to (A.1) which does not involve eigenvalues. If we let $\boldsymbol{U}$ and $\boldsymbol{V}$ be the left and right eigenvectors of $\boldsymbol{A}\left(t^{*}, t^{*}\right)$ then (A.1) is equivalent (see Note 2) to

$$
\boldsymbol{U} \frac{\partial}{\partial t} \boldsymbol{A}\left(t^{*}, t^{*}\right) \boldsymbol{V}=0
$$
For both $\boldsymbol{A}_{Q}$ and $\boldsymbol{A}_{W}$ we have $\boldsymbol{U}=(M, Q)$ and $\boldsymbol{V}=\left(\begin{array}{c}Q \\ M / 2\end{array}\right)$. With these
vectors in (A.2) we get

$$
\frac{Q^{\prime}}{Q}+\frac{M^{\prime}}{M}=0 \quad \text { (Queen control) }
$$

and

$$
\frac{3 Q^{\prime}}{Q}+\frac{M^{\prime}}{M}=0 \quad \text { (Worker control) }
$$

The first formula is the condition which maximises $Q M$; the second maximises $Q^{3} M$. 
Note 1.-These formulae remain valid for discrete overlapping generations at least in our case in which offspring mature in one year and each breeding adult, regardless of age, survives to breed next season with fixed probability $p$. Then if $x^{\prime}$ and $y^{\prime}$ denote number of reproductive offspring from $x$ and $y$ adults (queens and males respectively) and

$$
\left[\begin{array}{l}
x^{\prime} \\
y^{\prime}
\end{array}\right]=\boldsymbol{A}\left[\begin{array}{l}
x \\
y
\end{array}\right]
$$

the breeding population next season is

$$
\left[\begin{array}{l}
\bar{x} \\
\bar{y}
\end{array}\right]=(\boldsymbol{A}+p \boldsymbol{I})\left[\begin{array}{l}
x \\
y
\end{array}\right] .
$$

In this case the condition we need is (A.2) with $\boldsymbol{A}+p \boldsymbol{I}$ playing the role of $\boldsymbol{A}$. Since $\boldsymbol{A}+\psi=$ has the same eigenvectors as $\boldsymbol{A}$, and $p$ is independent of $t,($ A.2) is in fact unchanged.

Note 2.-Let $\boldsymbol{U}\left(t, t^{*}\right)$ and $\boldsymbol{V}\left(t, t^{*}\right)$ be the left and right dominant eigenvectors of $\boldsymbol{A}\left(t, t^{*}\right)$. Start with $(\boldsymbol{A}-\lambda \boldsymbol{I}) \boldsymbol{V}=0$ with everything evaluated at $\left(t, t^{*}\right)$. Differentiate with respect to $t$ and then evaluate at $t=t^{*}$. We get

$$
\left(\frac{\partial \boldsymbol{A}}{\partial t}-\frac{\partial \lambda}{\partial t}\right) \boldsymbol{V}+(\boldsymbol{A}-\lambda \boldsymbol{I}) \frac{\partial \boldsymbol{V}}{\partial t}=0 .
$$

Now apply $\boldsymbol{U}\left(t^{*}, t^{*}\right)$ at the left. We get

$$
\boldsymbol{U} \frac{\partial \boldsymbol{A}}{\partial t} \boldsymbol{V}-\frac{\partial \lambda}{\partial t} \boldsymbol{U} . \boldsymbol{V}=0
$$

since $\boldsymbol{U}(\boldsymbol{A}-\lambda \boldsymbol{I})=0$ at $\left(t^{*}, t^{*}\right)$. Hence (A.1) and (A.2) are equivalent. 